\title{
Efecto de la densidad poblacional en la productividad de caracoles (Helix aspersa) en un sistema a cielo abierto alimentados con acelga y suplemento alimentario balanceado
}

\author{
Olga González, Gladys Pérez Camargo, Macarena Membiela, \\ Diana Frezza, Norberto Bartoloni y Carlos Vieites
}

Facultad de Agronomía, Universidad de Buenos Aires. Ave. San Martín 4453. Buenos Aires, Argentina.

\begin{abstract}
O.M. González, G. Pérez Camargo, M. Membiela, D. Frezza, N. Bartoloni, and C. Vieites. 2008. Effect of population density on snail productivity (Helix aspersa) in an open sky system fed with Swiss chard and a balanced food supplement. Cien. Inv. Agr. 35(3):251257. Heliciculture is performed worldwide and has been promoted in Argentina; however, its development and expansion has been limited by several factors that make it impossible to reach the production volumes necessary for a commercial scale. Among other factors, it has been impossible to obtain a high amount of finished product according to the initial density determined in the fattening stage; additionally, difficulty in maintaining an adequate forage supply has limited snail production. In this study, the influence of snail density using balanced food supply on the final production was studied in an open sky system. Density had no significant effect on the mean final live weight. For snails cultured at densities higher than $200 \mathrm{snail} \cdot \mathrm{m}^{-2}$, the average diameter was lower than for snails cultured at $50 \mathrm{snail} \cdot \mathrm{m}^{-2}$ (low density); however, the difference between the moderate snail density $\left(100\right.$ snail $\left.\cdot \mathrm{m}^{-2}\right)$ and the low density was not significant. The highest productivity per unit of surface was about $510 \mathrm{~g} \cdot \mathrm{m}^{2}(\mathrm{n}=200)$, which is similar to the amount that heliciculture producers want to achieve and slightly lower than what has been reported previously.
\end{abstract}

Key words: Escargot, edible snail, Helix aspersa, heliciculture, snails, snail density, snail farming, snail farming.

\section{Introducción}

En Argentina, Helix aspersa Müller es la especie de caracol más ofrecida comercialmente. Se obtiene por recolección y es escasamente producido en cautiverio. La demanda proviene principalmente de consumidores de origen español e italiano (Arrébola et al., 2004; Arrébola y Alvarez, 2001).

Los sistemas de producción de caracol en cautiverio son a cielo abierto, a cielo cerrado o sistemas de producción mixtos (Arditi et al., 2003; Dignan y Obschatko, 2003; Marasco y Murciano, 2000). El sistema a cielo abierto

Recibido 30 octubre 2007. Aceptado 12 mayo 2008.

${ }^{1}$ Dirigir correspondencia a O. González: ogonzale@agro.uba.ar consiste en recintos al aire libre, en los que los caracoles habitan en las diferentes etapas de producción con vegetación implantada para tal fin (Sastre, 2006). Se estiman rendimientos de 10 tha ${ }^{-1}$ de caracoles (Sastre, 2006; San Román et al., 2004; Murphy, 2001).

Son varias las especies vegetales que se pueden implantar (Sastre, 2006; San Román et al., 2004; Laborda, 2003). No obstante, ello es motivo de controversia y al mismo tiempo no existe consenso respecto de la carga de caracoles, rotación de cultivos y formas de suplementación alimentaria. La suplementación se debería emplear principalmente en la etapa de terminación cuando la oferta forrajera no permita satisfacer los requerimientos de consumo (Membiela, 2007). 
En Argentina, la mayoría de los criaderos de caracoles son a cielo abierto (San Román et al., 2004; Laborda, 2003; Lagrifa, 2002). Cuando la disponibilidad de alimentos es escasa, en este sistema productivo se produce una concentración de caracoles alrededor de comederos y/o plantas. Esto conlleva un mayor riesgo sanitario debido a la alta densidad de caracoles (Perea et al., 2006). Eventualmente se producen muertes cuando el alimento es exclusivamente de origen vegetal y escaso (San Román et al., 2004).

En los recintos de reproducción, cría y terminación se suele sembrar Brassica oleraceae var. viridis, B. oleracea var. italica, Beta vulgaris var. cicla, Cichorium intybus y, menos frecuentemente B. napus y Helianthus annuus. Las distintas especies vegetales poseen diferentes requerimientos edafo-climáticos y tienen ciclos ontogénicos distintos, lo que determina una marcada heterogeneidad espacial y temporal en el recinto productivo, dificultando el manejo planta-caracol. Los caracoles muestran un comportamiento alimentario diferencial regido por la palatabilidad de las especies vegetales y por sus requerimientos nutricionales (Membiela, 2007; San Román et al., 2004).

En sistemas de producción a cielo abierto, la tendencia actual es brindar una cobertura vegetal para un mínimo consumo, que al mismo tiempo sirva de refugio, y suplementar con alimento balanceado o vegetales cultivados en otra huerta (Membiela, 2007; Sastre, 2006). Para un crecimiento óptimo se sugiere 10 a $16 \%$ de proteína cruda, $2 \%$ de extracto etéreo y un mínimo de 3 a $4 \%$ de calcio. Este último elemento lo obtienen del suelo o es suplementado (Thompson, 2005; Murphy, 2001; Dupont-Nivet et al., 2000).

En Argentina, la suplementación se hace comúnmente con alfalfa (Medicago sativa), girasol (H. annuus) o colza (B. napus), siendo poco habitual suplementar con alimento balanceado (San Román et al., 2004; Lagrifa, 2002). Especialistas españoles proponen que la cobertura vegetal sea poco palatable para que los caracoles la utilicen mayormente como refugio, y que la alimentación sea alimento balanceado suministrado ad libitum (Membiela, 2007; J. Arrébola, comunicación personal, 2006).

Es importante que los individuos dispongan de refugios naturales y artificiales. Así se reduce el riesgo de depredación y los protege del estrés ambiental (Lagrifa, 2002). Algunos expertos consideran conveniente proveer sólo refugios naturales, provenientes de la implantación de diferentes especies vegetales (Perea et al., 2006).

En sistemas de producción abiertos se recomienda una densidad de 200 caracoles $\cdot \mathrm{m}^{-1}$ en la fase de terminación. Para lograr esta población se debe considerar del 20 al 50\% de mortalidad y/o fugas en el proceso de la cría y engorde (San Román et al., 2004; Lagrifa, 2002). La densidad puede afectar el ciclo de vida de los moluscos terrestres en poblaciones naturales y en cautiverio (Mayoral et al., 2004; Dupont-Nivet et al., 2000; Cameron y Carter, 1979). Si la densidad es elevada, el crecimiento, la reproducción y el engorde se reducen o incluso se inhiben, aunque el alimento sea suficiente y de calidad. En estas condiciones es posible que feromonas, presentes en las secreciones naturales de los individuos, desencadenen la inhibición del ciclo productivo (Dupont-Nivet et al., 2000; Cameron y Carter, 1979).

Respecto al crecimiento y terminación en cautiverio, Cameron y Carter (1979) informan que en un período de $26 \mathrm{~d}$ se pueden detectar diferencias en grupos de juveniles y adultos de $H$. aspersa. La determinación del diámetro mayor de la valva es mejor indicador de crecimiento que el peso individual ya que éste último muestra gran variabilidad debido al grado de hidratación de los caracoles (Membiela, 2007; Lagrifa, 2002).

El exceso de humedad en los recintos de crianza produce conchas extremadamente blandas, con el consiguiente perjuicio en el embalaje y transporte para la venta. Además, en los ambientes excesivamente húmedos proliferan parásitos perjudiciales para la supervivencia de los moluscos de tierra. La presencia de planaria (gusanos planos, Clase Turbellaria, Filo Platelmintos) ha sido reportada en Argentina como un factor que ha originado el abandono 
de la producción de caracoles (Sastre, 2006; González et al., 2005, 2004). No existen técnicas de control que permitan eliminar este parásito sin afectar a los caracoles. En algunos criaderos se ha optado por provocar su muerte, disminuyendo drásticamente la humedad relativa de los recintos de producción. En estas condiciones y ante la desaparición de la cobertura vegetal por ausencia de agua, los caracoles cesan su actividad fisiológica (estivación o cese de actividad del verano). Este es un medio de defensa ante condiciones adversas. Para evitar la infestación se debería recurrir a recintos nuevos con cobertura vegetal para colocar los individuos que reanudarán su actividad en condiciones ambientales apropiadas. Si esto se debe realizar, la marcada estacionalidad de esta especie de caracoles y el tiempo requerido para el crecimiento de las plantas limitarán la posibilidad de terminar el producto en la época de mayor demanda (J. Rusinek, comunicación personal, 2005).

Los antecedentes de trabajos realizados con caracoles en otros países destacan la importancia de la cantidad de ejemplares sujetos a producción y de la disponibilidad de alimento en cantidad y calidad (Mayoral et al., 2004; Borja, 2003). La densidad poblacional muestra una relación inversa con el crecimiento y directa con la tasa de mortalidad (Mayoral et al., 2004; Cameron y Carter, 1979). La oferta de alimento adecuada se debe prever para todo el ciclo productivo (Thompson, 2005).

El objetivo del trabajo fue estudiar el efecto de la densidad poblacional sobre la productividad de caracoles en terminación en un sistema a cielo abierto utilizando acelga y alimento balanceado.

\section{Materiales y métodos}

\section{Condiciones de manejo}

Se montaron nueve cubículos, de 1 x 1 x $1 \mathrm{~m}$ cada uno, con tela de media sombra (50\%) en tres recintos productivos $(3 \times 13 \mathrm{~m})$ a cielo abierto, perimetrados con tela de media sombra, separados por calles y exteriormente cercados con alambrado olímpico. Se trasplantó acelga (B. vulgaris var. cicla) a $20 \times 20 \mathrm{~cm}$. La cobertura vegetal y tres tablas $(50 \times 15 \mathrm{~cm})$, colocadas en un ángulo de $45^{\circ}$ respecto del suelo, sirvieron como refugio (San Román et al., 2004; Lagrifa, 2002).

Para controlar especies vegetales silvestres y poder observar las fugas de caracoles, cada cubículo se circundó con un acolchado de polietileno negro (30 $\mu \mathrm{m}$ de espesor). Ante la eventual presencia de predadores (aves y roedores), los cubículos se protegieron con tela de media sombra en su parte superior y lateral. Para controlar roedores, se colocaron discos de warfarina (Brumoline ${ }^{\circledR}$ Arquim, Argentina), protegidos dentro de caños de cloruro polivinílico de $40 \mathrm{~cm}$ de largo.

Los cubículos se observaron diariamente al medio día para comprobar fugas y la presencia de predadores. Cada cubículo se regó diariamente, en la tarde o en la noche, por aspersión; la cantidad de agua suministrada fue la necesaria para mantener la cobertura vegetal.

En el momento de cosecha, se recolectaron primeramente los caracoles identificados a simple vista y los ubicados en los refugios artificiales, seguido por los ejemplares adheridos a la acelga. Posteriormente se removió el suelo para detectar ejemplares ubicados bajo la superficie del suelo.

\section{Población experimental}

Los caracoles se obtuvieron de un criadero comercial a cielo abierto en Las Perdices, Provincia de Entre Ríos, Argentina. Previo al inicio del ensayo, 7,5 $\mathrm{kg}$ de caracoles $(H$. aspersa) fueron purgados y sometidos durante $10 \mathrm{~d}$ a temperaturas decrecientes. De este modo se indujo el cese de las funciones vitales, propias de la primavera-verano. Con este propósito se mantuvieron durante $3 \mathrm{~d}$ a $20-22^{\circ} \mathrm{C}$, seguido por $3 \mathrm{~d}$ a $17-18^{\circ} \mathrm{C}$ y $4 \mathrm{~d}$ a $5^{\circ} \mathrm{C}$. Posteriormente, se realizó el proceso inverso, incrementando la temperatura en igual período de tiempo, hasta lograr que los caracoles retornaran a sus funciones vitales con un similar estado fisiológico intrapoblacional.

En cada cubículo se alojaron ( 8 de noviembre) caracoles sin peristoma reflejado (Lagrifa, 
2002), seleccionados al azar, de modo de obtener 50, 100 y 200 caracoles: $\mathrm{m}^{-2}$. El ensayo concluyó el 18 de diciembre luego de 40 d.

\section{Alimentación}

Se suplementó dos veces por semana con alimento para aves ponedoras en máximo de postura (®Ganave, Ganave Alimentos Balanceados, Argentina) compuesto por proteína (18\%), de extracto etéreo $(2,5 \%)$, fibra $(5 \%)$, calcio $(3,6 \%)$, fósforo $(0,5 \%)$, minerales totales (16\%) y $13 \%$ de humedad. El alimento se dispuso ad libitum en una bandeja descartable, de $25 \times 15 \mathrm{~cm}$, por cada cubículo. Las bandejas se retiraron durante las lluvias y luego se proporcionó nuevamente alimento fresco y seco. En recipientes plásticos $(10 \times 10 \mathrm{~cm})$ se colocaron esponjas embebidas en agua.

El consumo de alimento balanceado se evaluó entre el 16 de noviembre y el 11 de diciembre, estimando el porcentaje de materia seca del alimento balanceado suministrado y recolectado. También se estimó el consumo de la cobertura vegetal desde el inicio al final del ensayo, determinando la proporción de hojas de acelga consumida.

\section{Evaluación}

Para cada densidad inicial se evaluó el promedio y los desvíos estándares del diámetro individual inicial y final, el peso vivo total inicial y final, el incremento del diámetro, la productividad y la proporción de caracoles vivos, muertos y no logrados.

Se determinó la temperatura interior del cubículo al medio día y se registróla temperatura y humedad ambiental suministrada por el Servicio Meteorológico Nacional, Argentina.

\section{Diseño y análisis estadísticos}

Los caracoles se distribuyeron en forma completamente al azar en cada uno de los tres tratamientos (50, 100 y 200 caracoles $\cdot \mathrm{m}^{-2}$ ), con tres repeticiones. Se realizaron análisis de varianzanoparamétricos (prueba deKruskalWallis) y los promedios se compararon según comparación múltiple no paramétrica $(\mathrm{p}<0,05)$ con la ayuda del software InfoStat/Profesional (Facultad de Ciencias Agropecuarias, Estadística y Diseño, Universidad Nacional de Córdoba, Córdoba, Argentina).

\section{Resultados y discusión}

En el análisis de variancia para el peso vivo final, tomando como covariable el número final de caracoles logrados, no se detectaron diferencias significativas $(\mathrm{p}<0,05)$ entre los pesos vivos finales medios correspondientes a las tres densidades iniciales consideradas en este estudio (Cuadro 1).

Los desvíos estándar de los promedios del peso vivo total inicial fueron menores a los correspondientes al peso vivo total final. Ello indica que al comienzo del ensayo, los caracoles seleccionados tuvieron un peso semejante entre sí, a expensas de un estado fisiológico similar, resultante del purgado realizado inicialmente. Al concluir la experiencia los registros se realizaron al momento de ser recolectados, razón por la cual el diferente grado de hidratación y condición fisiológica resultaron en una mayor heterogeneidad. Debido a que el peso individual depende mayormente del grado de hidratación de los caracoles, no sería conveniente utilizarlo como un indicador del crecimiento (Membiela, 2007; Lagrifa, 2002).

Se utilizó el diámetro inicial como covariable para corregir la posible influencia que el diámetro inicial tuvo sobre el diámetro final. No obstante, a pesar de la fuerte influencia que tuvo el diámetro inicial sobre el diámetro final, las diferencias en diámetros finales medios obtenidos entre las tres densidades de caracoles fueron significativas. Por medio de comparaciones múltiples se obtuvo diferencias significativas $(\mathrm{p}<0,05)$ entre 200 caracoles $\cdot \mathrm{m}^{-2}$ (diámetro menor) y las restantes densidades. Las densidades de 50 y 100 caracoles $\cdot \mathrm{m}^{-2}$ mostraron mayor diámetro final, sin diferencias significativas entre sí. Cuando se analizó el incremento del diámetro individual, las diferencias obtenidas fueron significativas $(\mathrm{p}<0,05)$ entre 50 y 200 caracoles $\cdot \mathrm{m}^{-2}$. No hubo diferencias significativas entre la densidad menor y la media y entre ésta última y la mayor densidad. 
Cuadro 1. Efecto de la densidad poblacional en la producción de caracoles (Helix aspersa) en un sistema productivo a cielo abierto.

Table 1. Effect of population density on the production of snails (Helix aspersa) in an open sky system.

\begin{tabular}{|c|c|c|c|c|c|c|c|c|c|}
\hline \multirow{2}{*}{$\begin{array}{l}\text { Densidad } \\
\text { inicial } \\
\text { caracol } \cdot \mathrm{m}^{-2}\end{array}$} & \multicolumn{2}{|c|}{$\begin{array}{l}\text { Peso vivo total } \\
\mathrm{g}\end{array}$} & \multicolumn{3}{|c|}{$\begin{array}{l}\text { Diámetro }^{1} \\
\mathrm{~mm}\end{array}$} & \multirow[b]{2}{*}{$\begin{array}{c}\mathrm{P}^{1} \\
\mathrm{~g} \cdot \mathrm{m}^{-2}\end{array}$} & \multicolumn{3}{|c|}{ Individuos $^{1}$} \\
\hline & Inicial & Final & Inicial & Final & I & & $\begin{array}{l}\mathrm{V} \\
\%\end{array}$ & $\begin{array}{l}\mathrm{M} \\
\%\end{array}$ & $\begin{array}{c}\mathrm{NL} \\
\%\end{array}$ \\
\hline 50 & $\begin{array}{r}280,0 \\
\pm 5,0 \mathrm{~A}\end{array}$ & $\begin{array}{l}293,3 \\
\pm 1,9 \mathrm{~A}\end{array}$ & $\begin{array}{c}31,15 \\
\pm 3,98 \mathrm{~B} \text { a }\end{array}$ & 32,31 & $1,87 \mathrm{a}$ & $\begin{array}{c}13,3 \\
\pm 71,12\end{array}$ & $\begin{array}{c}67,3 \\
\pm 14,1 \mathrm{a}\end{array}$ & $\begin{array}{c}6,0 \\
\pm 2,0 \mathrm{a}\end{array}$ & $\begin{array}{c}26,7 \\
\pm 15,3 \mathrm{a}\end{array}$ \\
\hline 100 & $\begin{array}{r}552,5 \\
\pm 3,5 \mathrm{~A}\end{array}$ & $\begin{array}{c}570,0 \\
\pm 190,5 \mathrm{~A}\end{array}$ & $\begin{array}{l}31,39 \\
\pm 1,6 \mathrm{~A}\end{array}$ & $\begin{array}{c}32,42 \\
\pm 5,55 \mathrm{~B} \mathrm{a}\end{array}$ & $1,54 \mathrm{ac}$ & $\begin{array}{c}-5,0 \\
\pm 171,97\end{array}$ & $\begin{array}{c}56,3 \\
\pm 17,7 \mathrm{a}\end{array}$ & $\begin{array}{c}5,33 \\
\pm 2,3 \mathrm{a}\end{array}$ & $\begin{array}{r}38,3 \\
\pm 15,4 \mathrm{a}\end{array}$ \\
\hline 200 & $\begin{array}{l}1.212,7 \\
\pm 7,5 \mathrm{~A}\end{array}$ & $\begin{array}{c}1.476,0 \\
\pm 230,2 \mathrm{~A}\end{array}$ & $\begin{array}{r}30,75 \\
\pm 1,6 \mathrm{~A}\end{array}$ & $\begin{array}{c}31,64 \\
\pm 11,61 \mathrm{Bb}\end{array}$ & $1,05 \mathrm{bc}$ & $\begin{array}{c}264,0 \\
\pm 225,3\end{array}$ & $\begin{array}{c}66,3 \\
\pm 8,4 \mathrm{a}\end{array}$ & $\begin{array}{c}8,3 \\
\pm 6,1 \mathrm{a}\end{array}$ & $\begin{array}{c}29,5 \\
\pm 6,8 \mathrm{a}\end{array}$ \\
\hline
\end{tabular}

${ }^{1}$ Promedios de tres repeticiones ( \pm desvío estándar) del peso vivo total inicial y final, diámetro individual inicial y final, incremento del diámetro (I), productividad (P) y proporción de individuos vivos (V), muertos (M) y no logrados (NL).

${ }^{2}$ Promedios seguidos por iguales letras mayúsculas (filas) y minúsculas (columnas) no son estadísticamente diferentes entre sí a p $=0,05$. ${ }^{\prime}$ Means of three replicates ( \pm standard deviation) of initial and final number of live snails, initial and final individual diameter, diameter increment $(I))$, productivity $(P)$, and proportion of live specimens $(L)$ dead specimens $(D)$, and not obtained $(N O)$.

${ }^{2}$ Means followed by the same upper case (row) or lower case (column) letter are not statistically significant at $p=0.05$.

Con 50 caracoles $\cdot \mathrm{m}^{-2}$, el diámetro inicial promedio mostró mayor variabilidad, resultando ser más homogéneo en el diámetro final. En 200 caracoles $\cdot \mathrm{m}^{-2}$ se registró mayor homogeneidad en el diámetro inicial y menor diámetro final con mayor variabilidad. Esta situación se asimila a lo informado por Cameron y Carter (1979) y Dupont-Nivet et al. (2000), quienes destacaron que a mayor número de ejemplares por unidad de superficie la competencia entre individuos aumenta. Las reacciones dentro del grupo podrían provocar que los individuos presenten diferentes crecimientos. Es probable que en el tratamiento de menor densidad, hubiera menor competencia intraespecífica, razón por la cual los individuos mostraron mayor tamaño final y fueron más homogéneos.

No se detectaron diferencias significativas (5\%) para cada tratamiento en el porcentaje de caracoles vivos, muertos y no logrados. El promedio total de los muertos y de los no cosechados significó el $37 \% \pm 12.82$ del número inicial de caracoles. San Román et al. (2004) refieren que del 20 al $40 \%$ de los ejemplares no se logran en la etapa de cría en el sistema abierto. Según Lagrifa (2002) ocurre una disminución del $50 \%$ entre muertes y fugas desde la siembra de juveniles en los canteros hasta el fin del ciclo productivo.

La productividad promedio con una densidad de 200 caracoles $\cdot \mathrm{m}^{-2}$ fue de $264 \pm 225.3$ $\mathrm{g}$, equivalente a la reportada previamente por productores de diferentes regiones de Argentina (Vieites et al., 2007; González et al., 2005). Por ejemplo, en uno de esos tres recintos se cosecharon $510 \mathrm{~g} \cdot \mathrm{m}^{-2}$, similar a lo promocionado por el Grupo de Productores Helicícolas (Vieites et al., 2007). La variabilidad de los resultados obtenidos se pudo deber a las características biológicas propias de la especie y/o a su interacción con el sistema de producción, lo que debería ser confirmado por trabajos posteriores.

Las temperaturas más elevadas ocurrieron el 9 y 10 de diciembre, con 30,8 y $35,1^{\circ} \mathrm{C}$, respectivamente, coincidiendo con las menores humedades relativas (30 a 35\%). Para esos días dentro de los cubículos los registros térmicos fueron en promedio entre 5,5 y $8,2^{\circ} \mathrm{C}$ menores a los del exterior. Para el resto de las determinaciones la temperatura promedio en el interior de los cubículos fue, en la mayoría de los casos, menor a la del ambiente. La temperatura regula los ciclos de actividad de los moluscos, siendo la óptima entre 17 y $23^{\circ} \mathrm{C}$ (San Román et al., 2004). El máximo registro detectado en los cubículos al mediodía fue de $28,7^{\circ} \mathrm{C}$, momento en el que la mayoría de los caracoles permanecieron inactivos en los refugios artificiales, en las hojas o enterrados en el suelo. Este comportamiento coincidió 
con los antecedentes previamente reportados (Thompson, 2005; San Román et al., 2004; Laborda, 2003; Lagrifa, 2002).

En dos cubículos ( $\mathrm{N}^{\circ} 4$ y 7) fueron detectados unos pocos ejemplares de planaria, altamente nocivos para la supervivencia de los moluscos y cuya presencia se asocia con alta humedad relativa (J. Rusinek, entrevista personal, 2006). Durante el período de ensayo, el porcentaje de humedad relativa osciló entre 42 y $75 \%$, valores similares a los recomendados (San Román et al., 2004; Laborda, 2003). Sin embargo, la infestación, aunque escasa, en dos de los nueve cubículos abre un interrogante respecto a otros factores predisponentes, además de la humedad. Este tema deberá ser estudiado exhaustivamente, dados los trastornos que provoca en el sistema de producción a cielo abierto cuando ocurren severas infestaciones.

Se determinó por observación visual que el total de cobertura vegetal utilizada como fuente alimenticia osciló entre el 30 y $60 \%$. Si bien esta fue una estimación visual, permitió establecer que la oferta forrajera fue suficiente para un sistema abierto con suplementación con alimento balanceado.

Productores helicícolas argentinos, expresaron la imposibilidad de mantener un equilibrio entre la oferta y el consumo de forraje para la última etapa de producción en sistema de producción a cielo abierto (González et al., 2005, 2004). En el presente trabajo se comprobó que si se suplementa con alimento balanceado se puede superar este inconveniente, coincidiendo de este modo con resultados previos (Membiela, 2007).

Diez días antes de finalizar ensayo se detectó infestación con áfidos en plantas de acelga en los cubículos número $6(\mathrm{n}=50)$ y $8(\mathrm{n}=200)$. Sin embargo, en ésos cubículos las plantas libres de infestación brindaron la cobertura vegetal y la oferta de forraje adecuadas.

El consumo de alimento balanceado, expresado en materia seca, en un período de $20 \mathrm{~d}$ fue en promedio de $633,07 \pm 44,30 \mathrm{~g}$ para la densidad de 200 caracoles $\cdot \mathrm{m}^{-2}$; de 303,97 $\pm 108,49 \mathrm{~g}$ para 100 caracoles $\cdot \mathrm{m}^{-2} \mathrm{y}$ de 269,07 $\pm 92,72 \mathrm{~g}$ en 50 caracoles $\mathrm{m}^{-2}$. La variabilidad para cada tratamiento se pudo deber a que el alimento presentó en ciertas ocasiones exceso de humedad, a pesar de estar protegido para evitar la entrada de agua (lluvia o riego) en los comederos. Esa dificultad es propia del sistema empleado. No se han encontrado antecedentes que determinen el consumo de caracoles en terminación a cielo abierto suplementados con ración.

Sobre la base de los resultados obtenidos se concluye que los ejemplares de caracoles crecieron significativamente mejor con una densidad de 50 que con 200 caracoles $\cdot \mathrm{m}^{-2}$. Esta última densidad corresponde a lo comúnmente utilizado en un sistema abierto en la etapa de terminación de caracoles, en tanto la alimentación no sea un factor limitante.

\section{Resumen}

La helicicultura es una actividad practicada en diversas regiones del mundo que ha sido promocionada en Argentina en los últimos años. Sin embargo, su expansión se ha limitado por diversos factores que han impedido lograr una producción adecuada a escala comercial. Entre estos factores se destacan la imposibilidad de obtener una cantidad adecuada de producto terminado y la dificultad de mantener una oferta forrajera acorde con las necesidades. En el presente trabajo se estudió la influencia de la densidad de cría $\left(50,100\right.$ y 200 caracoles $\cdot \mathrm{m}^{-2}$ ) y la suplementación con alimento balanceado en la producción final de caracoles (Helix aspersa), para un sistema abierto y por unidad de superficie productiva. El peso vivo promedio final no mostró diferencias significativas para distintas densidades de cría. En la densidad mayor, 200 caracoles $\cdot \mathrm{m}^{-1}$, el diámetro individual fue significativamente menor respecto a 50 caracoles $\cdot \mathrm{m}^{-2}$ (densidad menor). No se encontraron diferencias significativas entre la densidad media (100 caracoles $\cdot \mathrm{m}^{-2}$ ) y la menor. La máxima productividad por unidad de superficie fue de $510 \mathrm{~g} \cdot \mathrm{m}^{-2}$ (un grupo original de $n=200$ ), cantidad similar a la que pretenden lograr algunos productores helicícolas argentinos y sensiblemente inferior a la extensamente publicitada $\left(1000 \mathrm{~g} \cdot \mathrm{m}^{-2}\right)$. 
Palabras clave: Alimentación, caracoles, densidad, helicicultura, producción en cautiverio.

\section{Agradecimientos}

Los autores agradecen al Profesor José R. Arrébola Burgos, Universidad de Sevilla, España, por la lectura crítica al presente trabajo y a Juan Rusinek, Las Perdices, Provincia de Entre Ríos, Argentina, por donar los ejemplares de caracoles empleados en este trabajo.

\section{Literatura citada}

Arditi, A., D. Rodríguez y R. Villar. 2003. Plan de negocios para la cría, procesamiento y comercialización de caracoles Helix aspersa. Tesina, Universidad Centro de Estudios Macroeconómicos. Buenos Aires, Argentina. $150 \mathrm{p}$.

Arrébola, J. y R. Alvarez. 2001. La explotación de los caracoles terrestres en España: aspectos biológicos y socioculturales. Temas de Antropología Aragonesa 11:139-172.

Arrébola, J., A Porrás, A. Cárcaba y A. Ruiz. 2004. Caracterización del sector helicícola andaluz: la captura de caracoles terrestres en Andalucía occidental. Sociedad Española de Malocología. Iberus (España) 22:31-41.

Borja, D. 2003. Estudio de prefactibilidad de la cría de escargot. Tesis de Grado. Carrera de Ciencias Agropecuarias. Escuela Superior Politécnica del Ejército. Facultad de Ciencias Agropecuarias Quito. Ecuador. 117 p.

Cameron, R. y M. Carter. 1979. Intra and interespecific effects of population density on growth and activity in some helicid land snails (Gastropoda: Pulmonata). Journal of Animal Ecology 48:237-246.

Dignan, V. y E. Obschatko. 2003. Panorama de las producciones agroalimentarias no tradicionales en la Argentina. Estudio 1.EG.33.7. Unidad de Preinversión (UNPRE). Banco Interamericano de Desarrollo. Buenos Aires. Argentina. 75 p.

Dupont-Nivet, M., V. Coste, P. Coinon, J.C. Bonnet y J.M. Blanc. 2000. Rearing density effect on the reproduction performance of the edible snail Helix aspersa Muller in indoor rearing. Ann. Zootech. 49: 447-456.

González, O.M., C.M. Vieites, y C.P Basso. 2004. Contribución al fortalecimiento del sistema helicícola en la República Argentina. www. agro.uba.ar/servicios/cpa/documento_final.pdf. (Consultado: agosto 2006).

González, O.M., C.P. Basso y C.M. Vieites. 2005.
Actividad helicícola en la Argentina: actualidad y acciones futuras. Rev. Facultad de Agronomía. Universidad de Buenos Aires. Buenos Aires, Argentina 25:83-91.

Laborda, G. 2003. Análisis de la Actividad de Cría de Caracoles Comestibles Terrestres. Disponible en 60 p. www.redagraria.com/ganaderia/ Caracoles/0_presentacion.html. (Consultado: agosto de 2004).

Lagrifa, L. 2002. Helicicultura. Cultivo del Caracol Terrestre. Editorial Propia. La Plata, Provincia de Buenos Aires, Argentina. 119 p.

Marasco, F. y C. Murciano. 2000. Guía Completa de la Cría de Caracoles. Editorial De Vecchi, S.A. Barcelona, España. 128 p.

Mayoral, A.G, A. Garcia, J. Perea, R. Martín, R. Martos, R. Acero y F. Peña. 2004. Efecto de la densidad de población sobre el tamaño del caracol Helix aspersa Müller. Archivos de Zootecnia 53:379-382.

Membiela, M. 2007. Efecto de la densidad de caracoles Helix aspersa Müller sobre la terminación y el comportamiento durante la fase de engorde en sistema abierto. Tesis de Grado para acceder al Título de Ingeniero Agrónomo. Facultad de Agronomía, Universidad de Buenos Aires. Buenos Aires, Argentina. 55p.

Murphy, B. 2001. Breeding and Growing Snails Commercially in Australia. RIRDC No. 00/188. http://www.rirdc.gov.au/reports/NAP/00-188 htm. (Consultado: mayo de 2005).

Perea, J., R. Martín, R. Acero, F. Goméz, A.M. García Mayoral, F. Peña y A. García. 2006. Selección de hábitat en caracoles terrestres y sus aplicaciones a la helicicultura. Archivos de Zootecnia 55R:1-12.

San Román, J., G. Giammarino y S. Vidal. 2004. Manual Helicícola: Cría de Caracoles a Cielo Abierto. Editorial Orientación Gráfica. Buenos Aires, Argentina. 175 p.

Sastre, R. 2006. Helicicultura. Cría de caracoles en la Argentina: una alternativa innovadora en agronegocios. Tesis de Maestría. Area Agronegocios y Alimentos. Facultad de Agronomía, Universidad de Buenos Aires, Argentina.139 p.

Thompson, R. 2005. Raising Snails. The alternative Farming Systems Information Center National Agricultural Library SRB 96-105. Disponible en: www.nalisda.gov/afsic/AFSIC_pubs/srb9605.htm. (Consultado: agosto de 2006).

Vieites, C.M., O.M. González y C. Acuña Seery. 2007. Producciones Animales Alternativas con Potencial de Desarrollo Mediato e Inmediato en la Argentina. Estudio 1.EE.152. Unidad de Preinversión (UNPRE). Banco Interamericano de Desarrollo. Editorial Fundación I+I. Buenos Aires, Argentina. 185 p. 